\title{
A INTERPRETAÇÃO EM SEMÂNTICA LINGUÍSTICA: UM PONTO DE PARTIDA IMAGINÁRIO ${ }^{1}$
}

\section{L'INTERPRÉTATION EN SÉMANTIQUE LINGUISTIQUE: UN POINT DE DÉPART IMAGINAIRE}

\author{
OSWALD DUCROT ${ }^{2}$
}

Tradução de Georges Sosthene Koman ${ }^{3}$ e Nayara Fernanda Dornas ${ }^{4}$

Coordenação e revisão:

André Stefferson Martins Stahlhauer ${ }^{5}$ e Soeli Maria Schreiber da Silva ${ }^{6}$

Revisão Técnica da Tradução:

Greciely Cristina da $\mathrm{Costa}^{7}$

O Linguista, especialmente o linguista semanticista, encontra-se diante do problema da interpretação numa situação que considero bastante particular. Isto, devido ao fato de que a interpretação para nós é um meio, um instrumento, enquanto as demais disciplinas a tomam como objeto. Algumas vezes é um objeto a constituir: tal é o caso para o historiador da filosofia que busca atribuir um sentido satisfatório a um texto, a seu ver, misterioso ou malentendido. Ou um objeto a explicar, como quando tentamos descobrir os procedimentos e os mecanismos que estão colocados em funcionamento na
Le linguiste, spécialement le linguiste sémanticien, se trouve, vis-à-vis du problème de l'interprétation, dans une situation que je crois assez particulière. Cela tient à ce que l'interprétation, pour nous, est un moyen, un instrument, alors que les autres disciplines qui ont affaire à elle la prennent pour objet. Quelquefois c'est un objet à constituer: ainsi pour l'historien de la philosophie qui cherche à attribuer un sens satisfaisant à un texte, à ses yeux, mystérieux ou mal compris. $\mathrm{Ou}$ bien un objet à expliquer, comme lorsqu'on tente de découvrir les processus et les mécanismes qui sont mis en œuvre en fait pour aboutir à des interprétations.

\footnotetext{
${ }^{1}$ Texto publicado originalmente na revista Confrontation, $\mathrm{n}^{\circ}$ 17, 119-130, 1987.

${ }^{2}$ Linguista francês (1930-), Diretor de Estudos na École des Hautes Études en Sciences Sociales (EHESS), em Paris, França. https://www.ehess.fr/fr/oswald-ducrot.

3 Doutorando em Linguística na Universidade Federal de São Carlos. \begin{tabular}{l} 
http://lattes.cnpq.br/5559926612678856. \\
\hline Mestranda em
\end{tabular}

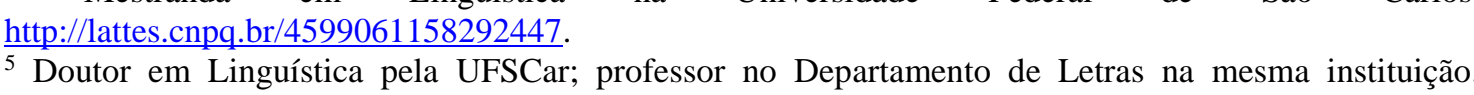
http://lattes.cnpq.br/1042124312764795.

${ }^{6}$ Doutora em Linguística pela Unicamp; professora no Programa de Pós-Graduação em Linguística da UFSCar. http://lattes.cnpq.br/8794598988783452.

7 Doutora em Linguística pela Unicamp; professora no Programa de Pós-Graduação em Ciências da Linguagem (UNIVÁS). http://lattes.cnpq.br/0290481380374492.
} 
verdade, para desembocar em interpretações. Ou ainda, um objeto a julgar, se temos como finalidade definir um conjunto de normas às quais uma "boa" interpretação deve satisfazer.

\section{É um papel metodológico muito diferente que desempenha a} interpretação em semântica linguística, pois somos obrigados a considerar as interpretações que a fala recebe na vida cotidiana como meios suscetíveis de nos ajudar a conhecer aquilo que é o nosso objeto próprio, a saber, a língua. Tomando como ponto de partida as interpretações dadas pelos ouvintes ${ }^{8}$ aos enunciados endereçados de locutores a alocutários, tentamos definir a partir dessas interpretações, o valor semântico do material linguístico que intervém nestes enunciados. $\mathrm{O}$ nosso ponto de partida é, assim, sempre do tipo: "tal locutor interpretou tal enunciado de tal maneira, outro tal locutor interpretou esse mesmo enunciado de outra maneira" (ao especificar que o locutor e o alocutário são eles mesmos "ouvintes" que interpretam o que dizem ou o que lhes é dito). E a nossa tarefa consiste em tentar descobrir, detrás dessas múltiplas interpretações, um valor semântico ligado às palavras empregadas, valor semântico que é o nosso próprio objeto, o qual devemos justificar a existência e a natureza ao mostrar que ele ajuda a compreender porque tais interpretações poderiam ser dadas ao enunciado e não outras.

Eis esquematizada o que é, a meu ver, a situação do linguista semanticista, e meu objetivo aqui é tomá-la com compaixão. Em primeiro lugar, mostrarei porque ela me parece inevitável, em seguida, indicarei alguns problemas que ela implica.
Ou encore un objet à juger, si l'on se donne pour but de définir un ensemble de normes auxquelles doit satisfaire une "bonne" interprétation.

C'est un rôle méthodologique bien différent que joue l'interprétation en sémantique linguistique. Car nous sommes obligés de considérer les interprétations que la parole reçoit dans la vie quotidienne comme des moyens susceptibles de nous aider à connaître ce qui est noire objet propre, à savoir la langue. Prenant pour point de départ les interprétations données par les auditeurs aux énoncés adressés par des locuteurs à des allocutaires, nous essayons, à partir d'elles, de définir la valeur sémantique du matériau linguistique intervenant dans ces énoncés. Notre point de départ est ainsi toujours du type: 'Tel auditeur a interprété tel énoncé de telle façon, tel autre a interprété ce même énoncé de telle autre façon" (en précisant que le locuteur et l'allocutaire sont eux-mêmes des "auditeurs", interprétant ce qu'ils disent ou ce qu'on leur dit). Et notre démarche consiste à essayer de découvrir, derrière ces multiples interprétations, une valeur sémantique attachée aux mots utilisés, valeur sémantique qui est notre objet propre, et dont nous devons justifier l'existence et la nature en montrant qu'elle aide à comprendre pourquoi telles interprétations ont pu être données à l'énoncé et non pas telles autres.

Voilà schématisée ce qui est, à mon avis, la situation du linguiste sémanticien, et mon objectif, ici, est de la faire prendre en compassion. D'abord je montrerai pourquoi elle me paraît inévitable, ensuite, j'indiquerai certains problèmes qu'elle implique.

\footnotetext{
${ }^{8}$ Nota dos tradutores: traduzimos auditeurs por ouvintes, que são aqueles que ouvem um discurso.
} 
Um pouco de terminologia me é necessário. Chamo "enunciado" um segmento de discurso, isto é, uma entidade que aparece num lugar e num momento dado e que não é, portanto, suscetível de ser repetido. Se eu disser a seguir "O tempo está bom. O tempo está bom", temos dois enunciados. A reflexão sobre a linguagem, ou, de qualquer modo, sobre as línguas, é o primeiro passo para decidir que diferentes enunciados podem ser a realização de uma mesma entidade abstrata, definida na gramática da língua e que chamo "frase". Em meu exemplo, grande parte dos gramáticos, ao menos todos aqueles que não atribuem à entonação o estatuto de entidade linguística fundamental, admitiriam que os dois enunciados em questão correspondem a uma única frase da língua francesa.

Do ponto de vista semântico, chamarei de "sentido" o valor semântico que um ouvinte (notadamente, conforme dito anteriormente, o locutor e o alocutário) atribui a um enunciado, em outros termos, à interpretação que lhe atribui um testemunho, independentemente se ele estiver fora do processo de comunicação ou envolvido nele, e, neste caso, se ele for ativo ou passivo. (Não insisto sobre os múltiplos problemas que suscitam essa terminologia, quando aplicada em situações efetivas. Por exemplo, tendo interpretado um enunciado no momento em que o ouvi, volto depois de um tempo à minha primeira interpretação, trata-se de duas interpretações de um mesmo enunciado ou de dois enunciados diferentes?). Quanto ao termo "significação", o reservo para o valor semântico da frase; tal como a frase, ela pertence então à língua e, enquanto tal, exerce um papel central em toda semântica linguística.
Un peu de terminologie m'est nécessaire. J'appelle "énoncé" un segment de discours, c'est-à-dire une entité apparaissant en un lieu et un moment donnés, et qui n'est donc pas susceptible de se répéter. Si je dis à la suite "Il fait beau. Il fait beau.", il y a deux énoncés. La réflexion sur le langage, ou, en tout cas, sur les langues, a pour premier pas de décider que différents énoncés peuvent être la réalisation d'une même entité abstraite, définie dans la grammaire de la langue, et que j'appelle "phrase". Dans mon exemple, la plupart des grammairiens, tous ceux, au moins, qui ne donnent pas à l'intonation le statut d'entité linguistique fondamentale, admettraient que les deux énoncés en question correspondent à une unique phrase de la langue française.

Du point de vue sémantique, j'appellerai "sens" la valeur sémantique qu'un auditeur (notamment, je l'ai dit, le locuteur et l'allocutaire) donne à un énoncé, en d'autres termes l'interprétation que lui attribue un témoin, qu'il soit extérieur au processus de communication ou y soit engagé, et, dans ce cas, qu'il y soit actif ou passif. (Je n'insiste pas sur les multiples problèmes que soulève cette terminologie lorsqu'on l'applique à des situations effectives. Par exemple, lorsqu'ayant interprété un énoncé au moment où je l'ai entendu, je reviens après coup sur ma première interprétation, s'agit'il de deux interprétations d'un même énoncé ou de deux énoncés différents ?) Quant au terme "signification", je le réserve pour la valeur sémantique de la phrase; comme la phrase, elle appartient donc à la langue, et, à ce titre, joue un rôle central dans toute sémantique linguistique. 
Armado dessas distinções, posso com mais clareza expor o problema esboçado há pouco. Eu dizia que a interpretação é para nós um meio e não um objeto. Na terminologia que acabo de apresentar, esta tese se torna: "Partimos dos sentidos dos enunciados e temos que estabelecer a partir deste ponto, as significações das frases". Tese essa que tem, notadamente, duas implicações. Por um lado, não nos contentamos em explicar porque uma frase tem a significação que ela tem: antes, temos que determinar qual é, para cada frase, sua significação. Em seguida, se chamamos "fato" ou "dado" o que o cada pesquisador considera como o ponto de partida de sua pesquisa (pois se deve estabelecer um bom ponto de partida), faz-se necessário admitir que os sentidos ou interpretações exercem o papel dos fatos para o linguista que os utiliza para constituir o seu objeto próprio, as significações.

Para mostrar estes dois pontos, preciso trazer à luz, de início, que a relação entre uma frase e sua significação não pode ter para o linguista o caráter de um fato, de um dado. Insisto sobre a especificação "para o linguista", pois seria muito fácil e, desinteressante, lembrar que a significação das frases é o produto de uma construção, e que ela não é dada por qualquer "intuição" que beneficiaria os detentores de uma língua. Espero que ninguém pense em negá-lo, o que equivaleria a acreditar que há fatos absolutos sem ver que o que serve de fato para $\mathrm{X}$ foi feito (i.e. produzido, construído) por Y, apenas pode ser um "dado" para X, o resultado do trabalho de Y. Não é este princípio epistemológico geral que vai ser questão aqui e não busco dar, sobre este ponto, uma ilustração suplementar à tese de Duhem
Armé de ces distinctions, je peux poser plus nettement le problème esquissé tout à l'heure. Je disais que l'interprétation est pour nous un moyen et non pas un objet. Dans la terminologie que je viens de présenter, cette thèse devient: "Nous partons des sens des énoncés, et nous avons à établir, à partir de là, les significations des phrases". Thèse qui a, notamment, deux implications. D'une part, nous ne nous contentons pas d'expliquer pourquoi une phrase a la signification qu'elle a: nous avons d'abord à déterminer quelle est, pour chaque phrase, sa signification. Ensuite, et si l'on appelle "fait" ou "donnée" ce que le chercheur considère comme le point de départ de sa recherche (car on doit bien partir), il faut admettre que les sens ou interprétations jouent pour le linguiste le rôle de faits, dont il se sert pour constituer son objet propre, les significations.

Pour montrer ces deux points, je dois faire voir que le rapport entre une phrase et sa signification ne peut pas avoir, pour le linguiste, le caractère d'un fait, d'une donnée, d'un point de départ. J'insiste sur la spécification "pour le linguiste". Car il serait trop facile, et sans intérêt, de rappeler que la signification des phrases est le produit d'une construction, qu'elle n'est pas donnée par quelque "intuition" dont bénéficieraient les détenteurs d'une langue. Personne, j'espère, ne songe à le nier, ce qui reviendrait à croire qu'il y a des faits absolus, sans voir que ce qui sert de fait à X a été lait (i.e. produit, construit) par Y: ne peut être une "donnée" pour X que le résultat du travail de Y. Ce n'est pas de ce principe épistémologique général qu'il va être question ici, et je ne cherche pas à donner, sur ce point, une illustration supplémentaire à la thèse de Duhem selon 
segundo a qual os fatos de hoje são construídos com as teorias de ontem.

O problema que me interessa é mais específico. Quero mostrar que a atribuição de uma significação às frases não pode ser considerada pelo linguista, atuando como linguista, enquanto o produto de um trabalho já feito pelos "usuários ingênuos" da língua, e que somente depois disso começaria o trabalho propriamente linguístico. Este último consistiria essencialmente em estabelecer os valores dos elementos que estruturam a frase (morfemas, construções sintáticas) e em descobrir as leis segundo as quais esses valores se combinam para constituir as significações das frases.

Se não posso aceitar essa concepção, é porque "os usuários ingênuos" nunca dizem nada da frase e só podem nos informar sobre sua interpretação dos enunciados. Talvez, ao pronunciar diante deles uma série de palavras ligadas segundo as regras da sintaxe e fora de todo contexto apropriado a um enunciado desta série, eles saberão dizer algo relacionado ao valor semântico dessa mesma série: eles poderão parafraseá-la, indicar o que seria compatível ou incompatível com ela, etc. Entretanto, ao analisar de mais perto este discurso sobre as séries de palavras fora de contexto (que não são, propriamente, nem frases, nem estruturas abstratas, nem enunciados produzidos por uma atividade de fala em relação ao contexto, mas algo como exemplos de gramática), percebe-se que tal consiste essencialmente em imaginar enunciados dessas séries numa situação banal e logo dizer como se interpretaria estes enunciados. Não se trata da frase que está, então, em questão, mas de um enunciado fictício. Antes do trabalho laquelle les faits d'aujourd'hui sont construits avec les théories d'hier.

Le problème qui m'intéresse est plus spécifique. Je veux montrer que l'attribution d'une signification aux phrases ne peut pas être considérée par le linguiste, exerçant son métier de linguiste, comme le produit d'un travail déjà fait par les "usagers naïfs" de la langue, et après lequel seulement commencerait le travail proprement linguistique. $\mathrm{Ce}$ dernier consisterait essentiellement à établir les valeurs des éléments' dont la phrase est faite (morphèmes, constructions syntaxiques) et à découvrir les lois selon lesquelles ces valeurs se combinent pour constituer les significations des phrases.

$\mathrm{Si}$ je ne peux pas accepter cette conception, c'est que les "usagers naïfs" ne disent jamais rien de la phrase et ne peuvent nous renseigner que sur leur interprétation des énoncés. Certes, si l'on prononce devant eux une suite de mots reliés selon les règles de la syntaxe, et cela en dehors de tout contexte approprié à un énoncé de cette suite, ils sauront dire quelque chose qui a rapport à la valeur sémantique de la suite elle-même: ils pourront la paraphraser, indiquer ce qui serait compatible ou incompatible avec elle, etc. Mais si on analyse de plus près ce discours sur les suites de mots hors contexte (qui ne sont à proprement parler ni des phrases, structures abstraites, ni des énoncés, produits par une activité de parole en rapport avec le contexte, mais quelque chose comme des exemples de grammaire), on s'aperçoit qu'il consiste essentiellement à imaginer des énoncés de ces suites dans une situation banale, et à dire comment on interpréterait alors ces énoncés. Ce n'est pas de la phrase qu'il est donc question, mais d'un énoncé fictif. Avant le travail linguistique il n'y a donc 
linguístico não há desse modo, não apenas nenhuma intuição (o que, conforme eu disse, me parece evidente), mas também nenhuma construção daquilo que poderia ser a significação da frase. Esta só pode, dessa maneira, ser um ponto de chegada e de nenhuma forma um ponto de partida (i.e. um dado, um fato) para a pesquisa ${ }^{9}$.

Contestar-se-á que a diferença entre linguistas e "usuários ingênuos" é menos clara do que eu supus. Ocorre, vão me dizer, que os próprios usuários fazem um trabalho de linguista e que diante de "um exemplo de gramática", não se contentam apenas em lhe atribuir um valor semântico, a um de seus enunciados num contexto trivial, mas operam uma forma de generalização a partir de uma pluralidade de enunciados desta série: eles buscam a intersecção dos sentidos desses diferentes enunciados e constroem, desse modo, um mínimo semântico comum que possa ser considerado como a significação da frase suposta subjacente à série. Não se pode sustentar, dessa maneira, que o trabalho daqueles usuários fornece para 0 linguista o dado de base, o fato a partir do qual ele pode empreender sua própria pesquisa.

Responderei que essa "linguística ordinária”, supondo que ela exista, forneceria, na verdade, significações desprovidas de todo valor explicativo. Se o linguista teórico as assumisse, se ele as escolhesse como fatos, ele se condenaria a nada poder dizer claramente sobre a atividade linguageira real. Tentar demonstrá-lo exigiria discutir detalhadamente pesquisas empíricas. Me contentarei em formular, do modo mais non seulement aucune intuition (ce qui, je l'ai dit, me semble aller de soi), mais aucune construction de ce qui pourrait être la signification de la phrase. Celle-ci ne peut ainsi être qu'un point d'arrivée, et en aucun cas un point de départ (i.e. une donnée, un fait) pour la recherche*.

On m'objectera que la différence entre linguistes et "usagers naïfs" est moins nette que je ne l'ai supposée. Il arrive, me dira-t-on, que les usagers fassent euxmêmes un travail de linguiste, et que, mis en présence d'un "exemple de grammaire", ils ne se contentent pas de lui attribuer, pour valeur sémantique, celle d'un de ses énoncés dans un contexte trivial, mais qu'ils opèrent une sorte de généralisation à partir d'une pluralité d'énoncés de cette suite: ils cherchent l'intersection des sens de ces différents énoncés, et construisent ainsi un minimum sémantique commun, qui peut être considéré comme la signification de la phrase censée sous-jacente à la suite. $\mathrm{Ne}$ peut-on pas soutenir alors que le travail de ces usagers fournit au linguiste la donnée de base, le fait à partir duquel il peut entreprendre sa propre recherche.

Je répondrai que cette "linguistique ordinaire", à supposer qu'elle existe, fournirait en fait des significations dépourvues de toute valeur explicative. $\mathrm{Si}$ le linguiste théoricien les reprenait à son compte, s'il les choisissait comme faits, il se condamnerait à ne rien pouvoir dire d'éclairant sur l'activité langagière réelle. Essayer de démontrer cela exigerait de discuter en détail des recherches empiriques. Je me contenterai de

\footnotetext{
${ }^{9}$ Cf. "L'expérience imaginaire" descrita na p. 107 (in: Dire et ne pas dire, Paris, Hermann, 1972). O que o presente capítulo acrescenta é que a experiência do sentido é tão "imaginária", apesar de ser incontornável, quanto a da significação.
} 
explícito possível, a ideia que deveria guiar a demonstração. $\mathrm{O}$ núcleo semântico comum que se encontraria em todos os enunciados seria, por definição, homogêneo ao sentido desses enunciados. Isso seria uma parte deste sentido - não longe do que entendemos comumente por sentido literal. E suporíamos que para cada enunciado particular se acrescentaria um aporte contextual ao núcleo semântico para constituir o sentido completo do enunciado. No entanto, as tentativas que buscam reconstituir aditivamente 0 sentido do enunciado me parecem de fato falhar. Vislumbra-se cada vez mais que a significação, para servir à explicação do sentido, deve ser de uma natureza diferente deste. Não pode ser de fato a situação do discurso em sua totalidade, ilimitada e inesgotável que intervém para constituir o sentido, mas apenas alguns de seus elementos. E a própria escolha desses elementos contextuais é parcialmente determinada pela frase. Esta deve, portanto, possuir nela algo que está necessariamente ausente do sentido, a saber, instruções, diretrizes que dizem quais informações é preciso buscar na situação, e como as explorar para interpretar os enunciados da frase. É, em todo caso, a uma conclusão desse tipo que G. Fauconnier ${ }^{10}$ ou eu mesmo, chegamos por itinerários completamente diferentes. Fauconnier fala em instruções para construir os diferentes espaços mentais cuja articulação constitui o sentido do enunciado; eu, da minha parte, falo em instruções para estabelecer os diferentes movimentos argumentativos ou ainda, vozes - cuja confrontação constitui o sentido, sendo este resultante de um tipo de diálogo cristalizado. Resumindo. Se a significação deve formuler, d'une façon aussi explicite que possible, l'idée qui devrait commander la démonstration. Le noyau sémantique commun qui se retrouverait dans tous les énoncés serait, par définition, homogène au sens de ces énoncés. Ce serait une partie de ce sens - pas très éloignée de ce que l'on entend d'habitude par sens littéral. Et l'on supposerait que, pour chaque énoncé particulier, un apport contextuel vient s'ajouter au noyau sémantique pour constituer le sens complet de l'énoncé. Or les tentatives cherchant à reconstituer additivement le sens de l'énonce me semblent en fait échouer. Il apparaît de plus en plus que la signification, pour servir à l'explication du sens, doit être d'une tout autre nature que lui. Ce ne peut pas être en effet la situation de discours dans sa totalité, illimitée et inépuisable, qui intervient pour constituer le sens, mais seulement certains de ses éléments. Et le choix de ces éléments contextuels est lui-même partiellement déterminé par la phrase. Celle-ci doit donc posséder en elle quelque chose qui est nécessairement absent du sens, à savoir des instructions, des directives disant quelles informations il faut chercher dans la situation, et comment les exploiter pour interpréter les énoncés de la phrase. C'est en tout cas à une conclusion de ce type que $\mathrm{C}$. Fauconnier ou moi-même sommes parvenus, par des voies tout à fait différentes. Fauconnier parle d'instructions pour construire les différents espaces mentaux dont l'articulation constitue le sens de l'énoncé; je parle, pour ma part, d'instructions pour mettre en place les différents mouvements argumentatifs - ou encore voix - dont la confrontation constitue le sens, celui-ci étant la résultante d'une

${ }^{10}$ G. Fauconnier, Espaces mentaux, Edition de Minuit, Paris, 1984. A ideia de instrução interpretativa presente na significação das frases é prefigurada pela "variável" de que é questão, por exemplo, aqui mesmo, p. 130 (in: Dire et ne pas dire, Paris, Hermann, 1972). 
explicar a construção do sentido a partir do contexto, ela deve conter muitos elementos que não podem aparecer no sentido uma vez constituído: o modo de uso do "meccano" não é parte integrante do objeto construído com o "meccano", o andaime não é, em princípio, parte integrante do prédio.

Um exemplo, que não visa a demonstrar, mas a fazer compreender o que eu quero dizer. Suponhamos que eu busco descrever o operador francês quase, tal como ele aparece em séries como "O dólar está quase 8 francos". Posso, ao assimilar esta série de palavras e a frase subjacente, perguntar para um informante o que ela quer dizer a seu ver. Sua primeira resposta será sem dúvida que ela atribui ao dólar um valor levemente inferior a $8 \mathrm{~F}$. Tal resposta ilustra a primeira atitude de que eu falei e que consiste em imaginar um enunciado da série numa situação padrão - entende-se por aí, a primeira situação que vem à mente, de acordo com as circunstâncias.

Para o informante que tem dado esta reposta, avisaremos que alguns enunciados da série podem de fato indicar um preço um pouco superior a 8F. (Este é, aliás, com efeito, o sentido que ela tinha quando a observei no $L e$ Monde, em outubro de 85). Para levar em conta essa objeção, meu informante talvez adote então a atitude que eu caracterizei como aquela da "linguística ordinária". Ele buscará o que é comum aos dois enunciados (aquilo que ele imaginou e aquilo do Le Monde). Ele dirá, por exemplo, que na frase estudada, quase significa "por volta de", essa aproximação podendo se situar tanto acima quanto abaixo. sorte de dialogue cristallisé. Je me résume. Si la signification doit expliquer la construction du sens à partir du contexte, elle doit contenir de nombreux éléments qui ne peuvent pas apparaître dans le sens une fois constitué: le mode d'emploi du meccano n'est pas partie intégrante de l'objet construit avec le meccano, l'échafaudage n'est pas, en principe, partie intégrante de l'immeuble.

Un exemple, destiné non pas à démontrer, mais à faire comprendre ce que je veux dire. Supposons que je cherche à décrire l'opérateur français presque, tel qu'il apparaît dans des suites comme "Le dollar est presque à 8 francs". Je peux, assimilant cette suite de mots et la phrase sous-jacente, demander à un informateur ce que, pour lui, elle veut dire. Sa première réponse sera sans doute qu'elle attribue au dollar une valeur légèrement inférieure à $8 \mathrm{~F}$. Une telle réponse illustre la première attitude dont j'ai parlé, et qui consiste à imaginer un énoncé de la suite dans une situation standard - en entendant par là la situation qui, étant donné les circonstances, vient la première à l'esprit.

A l'informateur ayant donné cette réponse, on fera remarquer que certains énoncés de la suite peuvent en fait indiquer un prix un peu supérieur à $8 \mathrm{~F}$. (c'est d'ailleurs le sens qu'elle avait lorsque je l'ai relevée dans Le Monde, en octobre 85). Pour tenir compte de cette objection, mon informateur adoptera peut-être alors l'attitude que j'ai caractérisée comme celle de la "linguistique ordinaire". Il cherchera ce qui est commun aux deux énoncés (celui qu'il a imaginé et celui du Monde). Il dira par exemple que presque, dans la phrase étudiée, signifie "aux environs de", ces environs pouvant se situer au-dessus comme au-dessous. 
Resta-me então mostrar que o "denominador comum" depreendido dessa forma não tem nenhum caráter explicativo. De um lado, ele conduz a previsões falsas que deixam entender que se pode dizer quase com o sentido de "mais ou menos" ou "aproximadamente", para assinalar que a realidade se situa "em torno" do valor mencionado. No entanto, me parece que não é, em hipótese alguma, o caso. Por outro lado, a resposta da "linguística ordinária" permeia um fato comprovado: se o locutor dá como objetivo ao seu enunciado, no movimento do discurso em que ele se situa, o de mostrar a cotação barata do objeto em questão (como no caso do título no Le Monde), quase $8 \mathrm{~F}$ indica um valor superior a $8 \mathrm{~F}$, embora, se a intenção argumentativa for contrária, o valor indicado por quase $8 \mathrm{~F}$ é inferior. A significação da frase contendo quase comporta então instruções dadas aos eventuais interpretadores dos enunciados: "Buscar se o locutor argumenta a partir da importância ou da fraqueza da quantidade de que ele fala (isto é, se sua conclusão seria melhor ou menos bem servida pela atribuição ao dólar de um valor superior ao invés de um valor inferior). No primeiro caso quase $8 \mathrm{~F}$ indica um preço inferior a $8 \mathrm{~F}$, no segundo, um preço superior". N.B. Uma apresentação mais detalhada, mostraria que a frase precisa se representar, uma vez extraídas as informações pertinentes da situação, um tipo de diálogo "colocado em cena"11 pelo locutor e cuja sinopse é dada pela frase. No caso do enunciado do Le Monde, um primeiro enunciador sustentaria que o valor do dólar é de $8 \mathrm{~F}$ e utilizaria esse fato para uma certa argumentação fundada sobre a cotação barata do dólar. Um segundo,
Il me reste donc à montrer que le "dénominateur commun" ainsi dégagé n'a aucun caractère explicatif. D'une part, il conduit à des prévisions fausses, laissant attendre qu'on puisse dire presque avec le sens de "plus ou moins" ou "approximativement", pour signaler que la réalité se situe "autour" de la valeur mentionnée. Or, il me semble que ce n'est jamais le cas. D'autre part, la réponse de la "linguistique ordinaire" passe à côté d'un fait avéré: si le locuteur donne comme but à son énoncé, dans le mouvement de discours où il se situe, de montrer le bon marché de l'objet en question (comme c'était le cas dans le titre du Monde), presque aleur $8 \mathrm{~F}$ indique une valeur supérieure, mais si l'intention argurnentative est contraire, la valeur indiquée par presque $8 \mathrm{~F}$ est inférieure. $\mathrm{La}$ signification de la phrase contenant presque comporte donc des instructions données aux interprétants éventuels des énoncés: "Chercher si le locuteur argumente à partir de l'importance ou de la faiblesse de la quantité dont il parle (c'est-à-dire si sa conclusion serait mieux ou moins bien servie par l'attribution au dollar d'une valeur supérieure que par celle d'une valeur inférieure). Dans le premier cas presque $8 \mathrm{~F}$ indique un prix inférieur à $8 \mathrm{~F}$, dans le second, un prix supérieur". N.B. Une présentation plus détaillée noterait que la phrase demande de se représenter, une fois extraites de la situation les informations pertinentes, une sorte de dialogue mis en scène par le locuteur et dont la phrase donne la synopsis. Dans le cas de l'énoncé du Monde, un premier énonciateur soutiendrait que la valeur du dollar est de $8 \mathrm{~F}$, et utiliserait ce fait pour une certaine argumentation fondée sur le bon marché du dollar. Un deuxième, à visée argurnentative contraire, donc fondée sur

11 N.T. Traduzimos Mise em scène por "colocado em cena" a fim de considerar a especificidade da expressão utilizada pelo autor. 
com vistas argumentativas contrárias, portanto, fundada sobre o custo elevado, lhe daria um valor superior a $8 \mathrm{~F}$. E um terceiro enunciador, que concorda sobre os fatos com o segundo, voltaria ao modo de argumentação do primeiro apresentando como insignificante a diferença entre $8 \mathrm{~F}$ e o valor real - tendo em vista o objeto da controvérsia. Enfim, a frase deveria assinalar que o locutor considera somente o ponto de vista do terceiro enunciador.

Se admitirmos de fato, conforme tentei sugerir, que a significação das frases não pode desempenhar para o linguista semanticista, o papel de fato, resta apenas o sentido dos enunciados ao qual podemos atribuir este papel. E para ir do sentido à significação, não vejo nenhuma outra possibilidade a não ser a de impor à significação uma função explicativa: ela deve ser enquanto tal, capaz de fazer compreender porque os enunciados de uma frase têm os sentidos que eles recebem na realidade da atividade da interpretação. Justificamos que tal frase tem tal significação, se a partir deste ponto, podemos imaginar um mecanismo que simula aquilo em meio ao qual os enunciados são efetivamente interpretados, quer dizer, que produz os mesmos resultados que este último. A interpretação aparece dessa forma para nós, como o meio de acessar o que é o nosso objeto próprio, a saber, a significação das frases.

A especificidade desta situação aparece, por exemplo, em comparação com a do jurista que estuda uma jurisprudência. $\mathrm{O}$ jurista toma como ponto de partida as interpretações aquelas dadas a um mesmo artigo de lei por diferentes juízes em situações diferentes. As interpretações que o artigo recebeu quando foi aplicado a essas la cherté, lui donnerait une valeur supérieure à $8 \mathrm{~F}$. Et un troisième énonciateur, d'accord sur les faits avec le second, reviendrait au mode d'argumentation du premier, en présentant comme négligeable la différence entre $8 \mathrm{~F}$ et la valeur réelle - vu l'objet de la controverse. La phrase devrait signaler enfin que le locuteur prend en charge seulement le point de vue du troisième énonciateur.

Si l'on veut bien admettre, comme j'ai essayé de le suggérer, que la signification des phrases ne peut pas jouer, pour le linguiste sémanticien, le rôle de fait, il ne reste guère que le sens des énoncés à quoi on puisse attribuer ce rôle. Et pour aller du sens à la signification, je ne vois guère d'autre possibilité que d'imposer à la signification une fonction explicative: elle doit être telle qu'elle puisse faire comprendre pourquoi les énoncés d'une phrase ont les sens qu'ils reçoivent en fait au terme de l'activité d'interprétation. Nous sommes justifiés de poser que telle phrase a telle signification si, à partir de là, nous pouvons imaginer un mécanisme simulant celui grâce auquel les énoncés sont effectivement interprétés, c'est-àdire produisant les mêmes résultats que ce dernier. L'interprétation apparaît bien ainsi, pour nous, comme le moyen d'accéder à ce qui est notre objet propre, à savoir la signification des phrases.

La spécificité de cette situation apparaît par exemple si on la compare à celle du juriste étudiant une jurisprudence. Le juriste prend pour point de départ des interprétations - celles que différents juges ont données, dans des situations différentes, à un même article de loi. Les interprétations que l'article a reçues lorsqu'il a été appliqué à ces situations 
situações correspondem, no paralelo que esboço, aos sentidos dados aos múltiplos enunciados da frase. Mas, é a este ponto que os paralelos divergem, o objetivo do jurista não é o de estabelecer o conteúdo do artigo a partir de suas interpretações. Este conteúdo, o jurista já conhece: ele o conhece na medida em que o artigo é escrito em francês e, se supõe que o jurista é capaz de compreendê-lo, assim como é capaz de compreender todo enunciado, e conhecer o contexto (o contexto permite ao jurista dar um sentido aos enunciados que compõem o artigo, é ao mesmo tempo o código em que se insere o artigo e a instituição jurídica para a qual o código é concebido). O linguista, ao contrário, não tem a princípio nenhum conhecimento da significação das frases (lembro que a significação corresponde na minha comparação àquilo que é para o linguista o conteúdo do artigo de lei). Mais precisamente, ele deve fingir ignorá-la. Para quem quiser abordar os problemas semânticos enquanto linguista, esse momento de ascetismo é com efeito um prévio: nossa « dúvida metódica » consiste em tentar esquecer o que podemos saber a respeito da língua (portanto, das frases) e em considerar apenas o sentido dos enunciados. Preciso fingir ignorar o que quer dizer a frase $\boldsymbol{O}$ dólar está quase $8 F$ - saber como se interpretam esses enunciados numa dada circunstância. E meu trabalho é o de inventar para a frase uma significação que dê conta das interpretações que eu constato.

Eis então, qual me parece ser a situação do linguista semanticista. Ele considera a interpretação ou o sentido como algo conhecido e reconstrói a partir deste material, significações supostamente desconhecidas. Resta mostrar o desconforto desta situação. Ele correspondent, dans le parallèle que j'esquisse, aux sens donnés aux multiples énoncés de la phrase. Mais, c'est là que les parallèles divergent, le but du juriste n'est pas d'établir le contenu de l'article à partir de ses interprétations. Ce contenu, le juriste le connaît déjà: il le connaît dans la mesure où l'article est écrit en français, et où le juriste est censé capable de le comprendre, comme il est capable de comprendre tout énoncé dont il connaît le contexte (le contexte permettant au juriste de donner un sens aux énoncés composant l'article, c'est à la fois le code dont l'article fait partie, et l'institution juridique pour laquelle le code est conçu). Le linguiste, au contraire, n'a au départ aucune connaissance de la signification des phrases (signification, je le rappelle, qui correspond, dans ma comparaison, à ce qu'est, pour le juriste, le contenu de l'article de loi). Plus exactement, il doit faire comme s'il l'ignorait. Pour qui veut aborder en linguiste les problèmes sémantiques, ce moment d'ascétisme est en effet un préalable: notre "doute méthodique" consiste à essayer d'oublier ce que nous pouvons savoir à propos de la langue (donc des phrases) et à considérer seulement le sens des énoncés. Je dois faire comme si j'ignorais ce que veut dire la phrase Le dollar est presque à $8 F$ - tout en sachant comment s'interprètent ses énoncés dans telle ou telle circonstance. Et mon travail est d'inventer pour la phrase une signification qui rende compte des interprétations que je constate.

Voilà donc quelle me semble la situation $\mathrm{du}$ linguiste sémanticien. Il prend l'interprétation ou le sens comme du connu et reconstruit à partir de ce matériau des significations supposées inconnues. Reste à montrer l'inconfort de cette situation. Il tient avant tout au statut 
confia antes de tudo no estatuto incerto dessas interpretações que nos servem de fatos, e no caráter torto das vias que levam a estas. Me contentarei em enumerar algumas hipóteses subjacentes à apreensão do sentido. Essas hipóteses, chamo-as "externas" entendendo por aí que elas são relativas às entidades das quais partimos a princípio (isto é, aos sentidos dos enunciados que constituem o nosso observável) e não aquelas que construímos (as significações das frases). Estas últimas são o objeto de outras hipóteses que chamo "internas".

Até este ponto, chamei "sentido" todo valor, exceto fonético e sintático, atribuído por um interpretante (o locutor, o alocutário ou um terceiro) a um enunciado particular. Mas em que consiste este valor? Deve-se entender por aí as ideias que atravessam o espírito de quem ouve ou pronuncia o enunciado no momento em que ele o ouve ou o pronuncia - quer dizer, aqueles que seguem o enunciado na mesma atividade linguística atual e que chamarei por convenção "o valor imediato"? Supondo até, que tal valor seja depreensível, vou tentar mostrar que ela não é de natureza a permitir a reconstrução da significação.

É preciso ver de fato que os "valores imediatos" surgidos no próprio exercício da linguagem não são certamente produzidos a partir das significações da frase. Não se sabe, certamente, muito sobre os mecanismos psicológicos que intervêm na compreensão espontânea dos enunciados, mas é claro que não há sucessão de duas etapas distintas em que a primeira consistiria em representar a significação da frase, e a segunda, em tentar reconstruir o valor do enunciado - incertain de ces interprétations qui nous tiennent lieu de faits, et au caractère tortueux des voies qui y conduisent. Je me contenterai d'énumérer quelques types d'hypothèses sous-jacentes à l'appréhension du sens. Ces hypothèses, je les appelle "externes", en entendant par là qu'elles sont relatives aux entités que nous nous donnons au départ (c'est-à-dire aux sens des énoncés, qui sont notre observable), et non à celles que nous construisons (les significations des phrases). Ces dernières font l'objet d'autres hypothèses, que j'appelle "internes".

Jusqu'ici, j'ai appelé "sens" toute valeur, autre que phonétique et syntaxique, attribuée par un interprétant (le locuteur, l'allocutaire ou un tiers) à un énoncé particulier. Mais en quoi consiste cette valeur? Faut-il entendre par là les idées qui viennent à l'esprit de celui qui entend ou prononce l'énoncé au moment où il l'entend ou le prononce - c'est-à-dire celles qui accompagnent l'énoncé dans le courant même de l'activité linguistique, et que j'appellerai par convention "la valeur immédiate"? En supposant même qu'une telle valeur soit cernable, je vais essayer de montrer quelle n'est pas de nature à permettre la reconstruction de la signification.

Il faut bien voir en effet que les "valeurs immédiates" surgies dans l'exercice même du langage ne sont certainement pas produites à partir des significations de la phrase. On ne sait certes pas grand chose des mécanismes psychologiques qui interviennent dans la compréhension spontanée des énoncés; mais il est clair qu'il n'y a pas succession de deux étapes distinctes dont la première consisterait à se représenter la signification de la phrase, et la deuxième, à essayer de re construire la valeur de l'énoncé, en 
ao explorar desta vez os dados contextuais. A abordagem efetiva deve ser mais caótica e basear-se, em grande parte, em analogias e oposições com enunciados já considerados ou ouvidos: o presente enunciado suscita, enquanto semelhante a estes, ideias e decisões que lhes tinham sido ligadas e, enquanto diferente, ideias e decisões opostas. O seu "valor imediato" é dessa forma determinado pelas evocações desencadeadas pela presença ou pela ausência nele de tal palavra ou grupo de palavras (como, de alguma forma, pela sua estrutura frásica global) - sem que ninguém possa, presentemente, revelar quais são as estratégias interpretativas utilizadas. Portanto, se por milagre, um linguista conseguisse, para um número suficiente de enunciados de uma frase, dar conta de seus valores imediatos a partir de uma significação geral atribuída a esta frase, isto comprovaria certamente sua habilidade, mas não conferiria nenhuma objetividade particular à escolha que ele fez desta significação: ele teria sem falha, simulado os mecanismos produtores dos valores imediatos, mas apesar disso, sem aprender nada sobre esses mecanismos.

As interpretações que tomaremos como fatos, não devem então, ser confundidas com os "valores imediatos" dos enunciados. Nós precisamos construí-las. E é a partir dessas construções semânticas relativas aos enunciados que poderemos construir as significações das frases. Antes de decidir "esta frase significa isto", é preciso primeiramente decidir "este enunciado da frase, interpretado por tal testemunha significa isto". O dramático seria o fato de que para estabelecer isto, seria preciso decidir a respeito daquilo. Provavelmente, nós teríamos adivinhado exploitant cette fois les données contextuelles. La démarche effective doit être beaucoup plus chaotique, et reposer en bonne partie sur des analogies et des oppositions avec des énoncés déjà envisagés ou entendus: l'énoncé présent suicite, en tant que semblable à eux, des idées et des décisions qu'on leur avait attachées, et, en tant que différent, des idées et des décisions opposées. La "valeur immédiate" qui est la sienne est ainsi largement déterminée par les évocations que déclenche, la présence ou. l'absence en lui de tel mot ou groupe de mots (autant, en tout cas, que par sa structure phrastique globale) - sans que personne, actuellement, puisse dire quelles sont les stratégies interprétatives utilisées. Si donc, par miracle, un linguiste arrivait, pour un nombre suffisant d'énoncés d'une phrase, à rendre compte de leurs valeurs immédiates à partir d'une signification générale attribuée à cette phrase, cela prouverait certes son habileté, mais ne confèrerait aucune objectivité particulière au choix qu'il a fait de cette signification: il aurait à coup sûr simulé les mécanismes producteurs des valeurs immédiates, mais sans rien nous apprendre pour autant sur ces mécanismes.

Les interprétations que nous prendrons pour faits ne doivent donc pus être confondues avec les "valeurs immédiates" des énoncés. Il nous faut les construire. El c'est à partir de ces constructions sémantiques relatives aux énoncés que nous pourrons construire les significations des phrases. Avant de décider "cette phrase signifie ceci", il faut d'abord décider "Cet énoncé de la phrase, interprété par tel témoin, signifie cela". Le dramatique serait que pour établir cela, il ail déjà fallu décider à propos de ceci. On a sans doute deviné que l'inconfort du sémanticien tient, pour moi, à ce cercle, 
que o desconforto do semanticista está ligado, a meu ver, a este círculo que esboçarei a partir de três considerações.

Notaremos em primeiro lugar que falta muito ao "valor imediato" para constituir uma interpretação utilizável no estabelecimento da significação. Testemunha, ativa ou passiva, de um enunciado, extrai-se dele, antes de tudo, todas as indicações semânticas "interessantes", aquelas que têm implicações para a prática. Se se trata por exemplo de um enunciado declarativo, vamos nos concentrar principalmente em informações que ele dá sobre a realidade, porque costumamos precisar das informações. E nem sequer a todas, aliás. Aquelas que já são sabidas, notadamente, têm mais chances de passarem despercebidas (se um amigo me diz "Meu carro está quebrado", reterei sem dúvida do seu enunciado a existência do fato de que o carro está quebrado e não o fato de que ele tem um carro). Consequência: atendo-se aos "valores imediatos", dever-se-ia por um lado admitir, conforme Austin tinha feito em sua primeira teoria, uma completa heterogeneidade entre os enunciados declarativos e os outros (interrogativos, imperativos etc.), que apenas parecem trazer indicações sobre $\mathrm{o}$ ato de linguagem realizado ao enunciá-los e, mais geralmente, sobre suas enunciações. E de outro lado, dentre os elementos informativos, aqueles que são implícitos ou, mais precisamente, pressupostos (a existência do carro no meu exemplo) apenas aparecerão em certos enunciados (quando, por exemplo, a frase "Meu carro está quebrado" é dita para alguém que ignora e descobre na mesma situação, a existência do carro).

Se se deseja evitar essas consequências, deve-se, para estabelecer que je vais esquisser à partir de trois remarques.

On notera d'abord qu'il manque beaucoup à la "valeur immédiate" pour constituer une interprétation utilisable dans l'établissement de la signification. Témoin, actif ou passif, d'un énoncé, on en tire avant tout les indications sémantiques "intéressantes", celles qui ont des implications pour la pratique. S'il s'agit par exemple d'un énoncé déclaratif, on fera attention avant tout aux informations qu'il donne concernant la réalité, parce que ce sont d'habitude les renseigne-ments dont on a besoin. Et même pas à toutes d'ailleurs. Celles que l'on connaît déjà, notamment, ont bien des chances de passer inaperçues (si un ami me dit "Ma voilure est en panne", je retiendrai sans doute de son énoncé l'existence de la panne, et non pas le fait qu'il a une voiture). Conséquence: à s'en tenir aux "valeurs immédiates", il faudrait d'une part admettre, comme avait fait Austin dans sa première théorie, une complète hétérogénéité entre les énoncés déclaratifs et les autres (interrogatifs, impératifs, etc.), qui semblent seuls apporter des indications sur l'acte de langage accompli en les énonçant et, plus généralement, sur leur énonciation. Et d'autre part, parmi les éléments informatifs, ceux qui sont implicites ou, plus précisément, présupposés (l'existence de la voiture dans mon exemple) n'apparaîtront que dans certains énoncés (lorsque, par exemple, la phrase "Ma voiture est en panne" est dite à quelqu'un qui ignore, et apprend par làmême, l'existence de la voiture).

Si l'on désire éviter ces conséquences, on devra, pour établir les interprétations, 
as interpretações, aceitar introduzir nelas alguns elementos não aparentes ao nível do "valor imediato", o que exige previamente que se tenha dado um esquema geral, uma grelha de interpretação. É o que faço, por minha vez, ao apontar que o sentido de um enunciado (tanto constativo, quanto performativo), e mesmo do enunciado, o mais tristemente informativo (o "está chovendo em Cherbourg" da previsão meteorológica) é uma descrição de sua enunciação, uma caraterização do evento que constitui sua aparição, caraterização no interior da qual se situam e, em que podem se deduzir, as indicações sobre a realidade. Desta maneira, desemboco em interpretações homogêneas para os performativos e os constativos. Eu aponto de outro lado que todo enunciado é polifônico, isto é, ele dá a fala, simultaneamente, para diferentes enunciadores que dizem coisas diferentes, ainda que o locutor se identifique e o alocutário se interesse apenas por alguns ou mesmo um só dentre eles (assim todos os enunciados de "Meu carro está quebrado" deixam entender ao mesmo tempo asserções que dizem respeito à existência do carro e de que ele está quebrado, embora seja levado em consideração primordialmente o "valor imediato" apenas de quebrado). Tal é o primeiro tipo de hipóteses externas que eu quis assinalar. Elas dizem respeito ao que se deve acrescentar ao "valor imediato" para colocá-lo num esquema geral do sentido ou da interpretação. Já de início, elas fazem aparecer o círculo no qual o semanticista está trancado. Dentre as motivações que o levam a homogeneizar as interpretações e a escolher tal esquema de homogeneização em vez de outro (aquelas que me fazem preferir por exemplo uma concepção enunciativa e accepter d'y introduire des éléments inapparents au niveau de la "valeur immédiate", ce qui exige qu'on se soit donné au préalable un schéma général, une grille d'interprétation. C'est ce que je fais, pour ma part, en posant que le sens d'un énoncé (aussi bien constatif que performatif), et même de l'énoncé le plus tristement informatif (le "il pleut à Cherbourg" du bulletin météorologique) est une description de son énonciation, une caractérisation de l'événement que constitue son apparition, caractérisation à l'intérieur de laquelle se situent, et dont peuvent se déduire, les indications sur la réalité. De cette façon, j'arrive à des interprétations homogènes pour les performatifs et les constatifs. Je pose d'autre part que tout énoncé est polyphonique, c'est-à-dire qu'il donne la parole simultanément à différents énonciateurs disant des choses différentes, même si le locuteur ne s'identifie et si l'allocutaire ne s'intéresse qu'à certains ou même un seul d'entre eux (ainsi tous les énoncés de "Ma voiture est en panne" font entendre à la fois des assertions portant sur l'existence de la voiture et sur celle de la panne, même si la plupart du temps, c'est la panne seule qui est prise en compte dans la "valeur immédiate"). Tel est le premier type d'hypothèses externes que je voulais signaler. Elles concernent ce qu'il faut ajouter à la "valeur immédiate" pour la faire entrer dans un schéma général du sens ou de l'interprétation. Déjà elles font apparaître le cercle où le sémanticien est enfermé. Parmi les motivations qui l'amènent à homogénéiser les interprétations, et choisir tel schéma d'homogénéisation plutôt qu'un autre (celles qui me font préférer par exemple une conception énonciative et polyphonique du sens* à une conception représentationnellc et monologique), il y a, entre autres choses, la décision 
polifônica do sentido ${ }^{12}$ a uma concepção representacional e monológica), existe, entre outras coisas, a decisão geral de ler frases atrás dos enunciados e, ao mesmo tempo, uma hipótese particular (interna) sobre a forma que devem tomar as significações. Nos alojamos nos enunciados que escolhemos "reencontrar" nas frases: a hipótese externa reflete então a hipótese interna.

A essas decisões motivadas por um defeito dos "valores imediatos", acrescentam-se aquelas motivadas por um excesso. Para obter interpretações que dão acesso à significação linguística, deve-se também, de fato subtrair aos valores imediatos, os enunciados. Suponhamos que T (=testemunha) ouve L (=locutor) dizer "Pedro vai chegar" (enunciado ao qual me refiro a $\mathrm{E}$ ). Diferentes razões podem fazer com que uma ideia I seja, para $\mathrm{T}$, associada ao enunciado. O problema é o de saber quais delas devem ser admitidas enquanto elementos da interpretação dada por $\mathrm{T}$ a E. Vários tipos de I podem ser distinguidos, por exemplo:

(1) T, no momento em que ele ouviu E, estava pensando em I, independentemente de E. Para caricaturar, podemos imaginar um I do tipo "Este meu sapato me machuca!". Vamos lembrar também o grave magistrado de que fala Pascal e cuja gravidade não impede, durante um sermão, de se interessar sobretudo no fato de que o predicador se esqueceu de fazer a barba.

(2) T se lembra, no momento em que ele ouve $\mathrm{E}$, de uma ideia que foi antes associada por ele, sobre o modo (1), a um enunciado análogo a $\mathrm{E}$. générale de lire des phrases derrière les énoncés, et en même temps, une hypothèse particulière (interne) sur la forme que doivent prendre les significations. On loge dans les énoncés ce que l'on a choisi de "retrouver" dans les phrases: l'hypothèse externe reflète alors l'hypothèse interne.

A ces décisions motivées par un défaut des "valeurs immédiates", s'ajoutent celles motivées par un excès. Pour obtenir des interprétations qui donnent accès à la signification linguistique, il faut aussi, en effet retrancher aux valeurs immédiates les énoncés. Supposons que T (-témoin) entend L (=locuteur) dire "Pierre va venir" (énoncé auquel je me réfère par E). Différentes raisons peuvent faire qu'une idée I soit, pour T, associée à l'énoncé. Le problème est de savoir lesquelles doivent cire admises comme éléments de l'interprétation donnée par $\mathrm{T}$ à $\mathrm{E}$. Plusieurs types de I peuvent être distingués, par exemple:

(1) $\mathrm{T}$, au moment où il a entendu $\mathrm{E}$, était en train de penser à I indépendamment de E. Pour caricaturer, on peut imaginer un I du genre "Ce que ma chaussure me fait mal !". Qu'on se rappelle aussi le grave magistrat dont parle Pascal, et dont la gravité ne l'empêche pas, pendant un sermon, de s'intéresser surtout au fait que le prédicateur a oublié de se raser.

(2) $\mathrm{T}$ se rappelle, au moment où il entend $\mathrm{E}$, une idée qui a été autrefois associée pour lui, sur le mode (1), à un énoncé analogue à $\mathrm{E}$.

${ }^{12}$ O. Ducrot, Le dire et le dit, Editions de Minuit, Paris, 1984, chap. 8. 
(3) T, ao ouvir E, deduz I (I pode ser por exemplo a ausência de João à reunião em questão - T sabendo que Pedro e João se evitam cuidadosamente - ou $\mathrm{T}$, raciocinando a partir da própria enunciação de $\mathrm{L}$, conclui que $\mathrm{L}$ conhece Pedro, se interessa por ele, deseja falar dele, etc.)

(4) T supõe que L, ao dizer E, queria que ele soubesse I. Um filósofo griceano distinguiria de outro modo em (4) diferentes sub-possibilidades, entre outras:

(4a) I é o que, segundo T, L quis abertamente deixar saber, isto é, o que ele quis deixar saber ao deixar saber por isto que ele tinha a intenção de deixá-lo saber.

(4b) I é o que L quis deixar saber, sem querer, para que esta intenção seja adivinhada.

Não devo aqui buscar quais, dentre os diferentes componentes do valor imediato, entram ou não entram no que chamarei "interpretação de E por T". $\mathrm{O}$ que quero trazer à luz, é que a introdução de um desses elementos no (ou sua exclusão fora do) objeto "interpretação", concebido não enquanto um emaranhado acidental, mas como uma entidade estruturada; esta admissão ou esta exclusão, dependem da finalidade atribuída ao conceito de interpretação. De acordo como que se quer fazer com as interpretações de enunciados, serão definidas para elas, tal ou tal limite, sem que haja, no mesmo interior dos valores imediatos, um traço intrínseco que os denuncia como fazendo parte ou não do sentido do enunciado. Porém, a situação do linguista tal como a apresentei, fornecerá um critério para operar a triagem. Se fixamos como papel dos
(3) $\mathrm{T}$, en entendant $\mathrm{E}$, déduit I (I peut être par exemple l'absence de Jean à la réunion en question - $\mathrm{T}$ sachant que Pierre et Jean s'évitent soigneusement -ou bien $\mathrm{T}$, raisonnant à partir de l'énonciation même de $\mathrm{L}$, en conclut que $\mathrm{L}$ connaît Pierre, s'intéresse à lui, désire parler de lui, etc.)

(4) $\mathrm{T}$ suppose que $\mathrm{L}$, en disant $\mathrm{E}$, voulait lui faire savoir I. Un philosophe gricéen distinguerait d'ailleurs dans (4) différentes sous-possibilités, entre autres:

(4a) I est ce que, selon $\mathrm{T}$, $\mathrm{L}$ voulait ouvertement faire savoir, c'est-à-dire ce qu'il voulait faire savoir en faisant savoir pour cela qu'il avait l'intention de le faire savoir.

(4b) I est ce que $L$ voulait faire savoir sans vouloir pour autant que l'on devine cette intention.

Je n'ai pas ici à chercher lesquels, parmi les différents composants de la valeur immédiate, entrent ou n'entrent pas dans ce que j'appellerai "interprétation de E par T'. Ce que je veux faire voir, c'est que l'introduction d'un de ces éléments dans (ou son exclusion hors de) l'objet "interprétation", conçu non comme un ramassis accidentel, mais comme une entité structurée, cette admission ou cette exclusion dépendent de la finalité attribuée au concept d'interprétation. Selon ce que l'on veut faire avec les interprétations d'énoncés, on leur fixera telle ou telle limite, sans qu'il y ait, à l'intérieur même des valeurs immédiates, un trait intrinsèque qui les dénonce comme faisant ou non partie du sens de l'énoncé. Or la situation de linguiste telle que je l'ai présentée, fournira un critère pour opérer le tri. Si on fixe pour rôle au sens de l'énoncé de donner accès à la 
enunciados dar acesso à significação da frase, excluiremos do sentido tudo o que não é, diretamente ou indiretamente, e senão completamente, ao menos parcialmente, explicável a partir da significação.

O que faz aparecer uma segunda vez o círculo que é a nossa prisão. Pois, de acordo com as nossas hipóteses (internas) sobre a significação de uma frase, nos tornamos capazes, ou incapazes, de compreender tal ou tal elemento eventual do sentido, elemento que incluiremos no sentido, num caso, e excluiremos no outro. Essas interpretações do enunciado que servem para constituir o valor semântico da frase, não podemos, por conseguinte, delimitá-las independentemente deste valor. O exemplo de quase o mostra imediatamente. Será que a intenção de mostrar, seja a cotação barata, seja o custo elevado do dólar, faz parte do sentido dos enunciados "O dólar está quase 8 francos"? Muitos dos linguistas responderiam não, e dariam como razão que tal elemento de interpretação não teria relação com a frase utilizada - frase suscetível de servir tanto a uma intenção quanto a outra. Mas, suponhamos que se admita a descrição de quase proposta há pouco. Neste caso, a frase, se for verdadeiro que ela não prejudica a intenção, requer que se considere esta intenção, e se calcule a partir dela se o enunciado coloca o dólar acima ou abaixo de oito francos. A intenção argumentativa entra então, tendo em vista o nosso objeto, quando delimitamos o mesmo sentido do enunciado: ela não é mais, em relação ao enunciado, marginal, mas central. Neste ponto, o sentido ainda nos interessa, no exercício da nossa profissão, somente na medida em que para explicá-lo, temos a justificativa para construir significações de frase: reduz-se signification de la phrase, on exclura du sens tout ce qui n'est pas, directement ou indirectement, et sinon complètement, au moins partiellement, explicable à partir de la signification.

Ce qui fait apparaître une seconde fois le cercle qui est notre prison. Car, selon nos hypothèses (internes) sur la signification d'une phrase, nous deviendrons capables, ou incapables, de rendre compte de tel ou tel élément éventuel du sens, éléments que nous inclurons dans le sens, dans un cas, et exclurons dans l'autre. Ces interprétations de l'énoncé qui nous servent à constituer la valeur sémantique de la phrase, nous ne pouvons donc pas les délimiter indépendam-ment de cette valeur. L'exemple de presque le montre immédiatement. Est-ce que l'intention de montrer soit le bon marché soit la cherté du dollar fait partie du sens des énoncés "Le dollar est presque à huit francs"? Beaucoup de linguistes répondraient non, et donneraient comme raison qu'un tel élément d'interprétation serait sans rapport avec la phrase utilisée - phrase susceptible de servir une intention comme l'autre. Mais supposons qu'on admette la description de presque proposée tout à l'heure. Dans ce cas, la phrase, s'il est vrai qu'elle ne préjuge pas de l'intention, demande de prendre en compte cette intention, et de calculer à partir d'elle si l'énoncé place le dollar en dessus ou au dessous de huit francs. L'intention argumenlative entre alors, vu l'objet qui est le nôtre lorsque nous délimitons le sens, dans le sens même de l'énoncé: elle n'est plus, par rapport à l'énoncé, marginale, mais centrale. Ici encore, le sens nous intéresse, dans l'exercice de noue métier, dans la mesure seulement où, pour l'expliquer, nous sommes justifiés de construire des significations de phrase: il se réduit donc, par définition, 
assim, por definição, ao que podemos explicar fazendo uso dessas significações.

O último tipo de hipóteses externas de que falarei não diz mais respeito ao excesso ou ao defeito do sentido em relação ao "valor imediato", mas à configuração intrínseca do sentido, à articulação desses diferentes componentes. Tornou-se banal dizer que o sentido de um enunciado comporta em si mesmo indicações do tipo retórico. Dito de outro modo: há certos elementos do sentido que são ligados à ideia de que são obtidos por metáfora, metonímia, lítote, ...etc. -sendo sua origem constitutiva de seu status. Um enunciado como "Esse cara é uma galinha molhada"13 não significa somente que a pessoa em questão é medrosa, incapaz de assumir riscos..., mas indica também que esta caracterização é obtida por analogia com o comportamento de um certo animal numa determinada situação (e isto, mesmo se, como no meu caso, admito, apenas conhecemos na verdade o comportamento dos covardes por analogia com os "traços de caráter" emprestados às pessoas acusadas de serem parecidas com eles). Vou tentar mostrar que a atribuição a um enunciado de tal movimento retórico interno, mais do que em outro, é necessariamente ligado a uma concepção da significação, e reciprocamente.

O exemplo de lítotes mais banal, nos tratados franceses de retórica, é talvez o "Vá, eu não te odeio" de Chimena para Rodrigo no ato 3 do Cid (Sc. 4). Este enunciado é geralmente apresentado como uma declaração de amor, mas uma declaração feita por pudor ou por preocupação com o decoro à ce que nous pouvons expliquer en nous servant de ces significations.

Le dernier type d'hypothèses externes dont je parlerai ne concerne plus l'excès ou le défaut du sens par rapport à la "valeur immédiate", mais la configuration intrinsèque du sens, l'articulation de ses différents composants. Il est devenu banal de dire que le sens d'un énoncé comporte en luimême des indications de type rhétorique. Autrement dit: à certains éléments du sens est attachée l'idée qu'ils sont obtenus par métaphore, métonymie, litote, ...etc. leur origine étant constitutive de leur statut. Un énoncé de "Ce type est une poule mouillée" ne signifie pas seulement que la personne en question est craintive, incapable de prendre des risques..., mais indique aussi que cette caractérisation est obtenue par analogie avec le comportement d'un certain animal dans une certaine situation (et cela même si, comme c'est, je l'avoue, mon cas, on ne connaît en fait le comportement des poules mouillées que par analogie avec le "trait de caractère" prêté aux personnes accusées de leur ressembler). Je vais essayer de montrer que l'attribution à un énoncé de tel mouvement rhétorique interne plutôt que tel autre est nécessairement lié à une conception de la signification, et réciproquement.

L'exemple de litote le plus rebattu dans les traités français de rhétorique est sans doute le "Va, je ne te hais point" de Chimène à Rodrigue à l'acte 3 du Cid (Sc. 4). On présente généralement cet énoncé comme une déclaration d'amour, mais une déclaration formulée, par pudeur ou souci des convenances, sous forme

13 "Ce type est une poule mouillée". Nota dos tradutores: a expressão "uma galinha molhada" pode ser traduzida por "covarde", em português. 
sob forma litótica. Admitimos então, na interpretação do enunciado, ao mesmo tempo, um "valor literal" que seria fraco, e um "valor figurado" forte, obtido retoricamente a partir da primeira, e enfim, o próprio recurso à lítotes, recurso este que também é um componente do sentido - e essencial para definir a personagem de Chimena e, mais geralmente, o herói corneliano lutando, segundo a imagem tradicional, para ser quem ele deve ser a despeito de quem ele é. Se admitimos essa interpretação do enunciado, seremos levados a considerar o valor literal fraco atribuído ao enunciado de Chimena, a uma concepção linguística muito particular da negação. Diremos que a negação de um predicado P constitui um predicado oposto P', que se $\mathrm{P}$ é do tipo semântico positivo (cf. amar), a negação (não amar) é forte, mas se $\mathrm{P}$ é do tipo semântico negativo (cf. odiar), a negação (não odiar) é fraca. Uma teoria linguística da negação como esta, explica, com efeito, que as palavras de Chimena têm, a um primeiro nível, um valor fraco ("literal", num sentido em que ela deriva diretamente da frase), e que se precisa de uma lítotes para extrair delas um valor figurado forte ${ }^{14}$.

Notaremos, contudo, que uma interpretação diferente é completamente possível (me foi relatado por Christian Plantin, para quem eu devo por ter visto o caráter mais arbitrário da interpretação litótica imposta pela tradição escolar). Ela consiste em compreender "Eu não odeio você" como não indicando nada mais do que a falsidade do enunciado assertivo correspondente ("Eu te odeio"). $\mathrm{Na}$ medida em que os lugares comuns psicológicos e o código moral que compõem a verossímil teatral exigem que Chimena odeie o assassino de seu pai, a simples inexistência desse ódio tem litotique. On admet donc, dans l'interprétation de l'énoncé, à la fois une "valeur littérale" qui serait faible, une "valeur figurée", forte, obtenue rhéloriqucmeni à partir de la première, et enfin le recours même à la litote, recours qui est lui aussi un composant du sens et essentiel pour définir le personnage de Chimène, et, plus généralement, le héros cornélien luttant, selon l'image traditionnelle, pour être ce qu'il doit être malgré ce qu'il est. Si on admet cette interprétation de l'énoncé, on sera amené, pour rendre compte de la valeur littérale faible attribuée à l'énoncé de Chimène, à une conception linguistique bien particulière de la négation. On dira que la négation d'un prédicat $\mathrm{P}$ constitue un prédicat opposé $\mathrm{P}$ ', que si $\mathrm{P}$ est de type sémantique positif (cf. aimer), la négation (ne pas aimer) est forte, mais que si $\mathrm{P}$ est de type sémantique négatif (cf. haïr), la négation (ne pas haïr) est faible. Une telle théorie linguistique de la négation explique en effet que les paroles de Chimène aient, à un premier niveau, une valeur faible ("littérale", en ce sens qu'elle est issue directement de la phrase), et qu'il faille une litote pour en tirer une valeur figurée forte.

On notera, pourtant qu'une interprétation différente est tout à fait possible (elle m'a été signalée par Christian Plantin, à qui je dois d'avoir vu le caractère au mieux arbitraire de l'interprétation litotique imposée par la tradition scolaire). Elle consiste à comprendre "Je ne te hais point" comme n'indiquant rien d'autre que la fausseté de l'énoncé assertif correspondant ("je te hais"). Dans la mesure où les lieux communs psychologiques et le code moral qui composent le vraisemblable théâtral exigent que Chimène haïsse le meurtrier de son père, la simple inexistence de cette

${ }^{14}$ Esta representação da negação é proposta no capítulo 4, p. 137-139, da obra Le dire et ne pas dire. 
por ela própria um valor muito forte para acionar uma lítotes. Se acontece, além disso, de que a réplica de Chimena pode ser interpretada como sinal de amor, e não como declaração, nem como "literal" e nem figurado, é porque só o excesso de amor pode levar o herói corneliano a ultrapassar o dever (como parece atestálo, na esteira da cena, o "O milagre de amor" de Rodrigo). Suponhamos que se admita uma certa interpretação do verso de Corneille; o linguista encontrará nela uma justificação para descrever a frase negativa de uma maneira completamente diferente daquela à qual conduzia a interpretação tradicional. É para uma concepção "polifônica" da negação que vamos ser conduzidos. A frase negativa, irão dizer, serve para assinalar a presença e o debate de dois enunciadores antagonistas: a frase $E u$ não te odeio exige assim, por meio da sua significação intrínseca, que se entenda em cada um de seus enunciados, uma voz que expressa o ódio, e uma outra que se opõe à primeira. $\mathrm{Na}$ interpretação de um enunciado, devese colocar, adicionalmente, a uma significação particular, as duas vozes, assimiladas a personagens diversos. No caso do $\mathrm{Cid}$, o enunciador que expressa o ódio poderia se identificar à voz do dever, o enunciador que o rejeita, sendo identificado como o locutor, quer dizer, à Chimena, que inventa uma generosidade de amor que contradiz o dever ordinário, e, portanto, o cumpre em um nível mais elevado - o que é o milagre corneliano.

Até aqui, minha análise apenas mostrou - supondo que seja aceito - que a escolha do linguista (escolha que diz respeito à frase e à significação, portanto as hipóteses internas) se baseiam em uma decisão prévia do retórico (que se situa, por sua vez, ao nível do enunciado e do sentido, portanto das hipóteses externas). haine a par elle-même une valeur trop forte pour déclencher une litote. S'il se trouve de surcroît que la réplique de Chimène peut être interprétée comme marque d'amour, ce n'est pas en tant que déclaration, ni "littérale" ni figurée, c'est parce que seul l'excès d'amour peut entraîner le héros cornélien à dépasser le devoir (comme semble l'attester, dans la suite de la scène, le "O miracle d'amour" de Rodrigue). Supposons qu'on admette une telle interprétation du vers de Corneille; le linguiste y trouvera une justification pour décrire la phrase négative d'une façon tout à fait différente de celle à laquelle menait l'interprétation traditionnelle. C'est à une conception "polyphonique" de la négation qu'on va être conduit. La phrase négative, dira-ton, sert à signaler la présence et le débat de deux énonciateurs antagonistes: la phrase Je ne le hais point exige ainsi, de par sa signification intrinsèque, que l'on entende, dans chacun de ses énoncés, une voix qui exprime la haine, et une autre qui s'oppose à la première. Pour l'interprétation d'un énoncé, il faudra procéder en plus à une identification particulière des deux voix, assimilées à des personnages divers. Dans le cas du Cid, l'énonciateur qui exprime la haine pourrait s'identifier à la voix du devoir, l'énonciateur qui la rejette étant identifié au locuteur, c'est-à-dire à Chimène, inventant une générosité d'amour qui contredit le devoir ordinaire, et par là même l'accomplit à un niveau plus élevé - ce qui esi le miracle cornélien.

Jusqu'ici, mon analyse a seulement montré - à supposer qu'on l'admette - que le choix du linguiste (choix qui concerne la phrase et la signification, donc les hypothèses internes) se fonde sur une décision préalable du rhétoricien (qui se situe, elle, au niveau de l'énoncé et du sens, donc des hypothèses externes). $\mathrm{Ce}$ 
Isto é apenas a primeira metade do círculo. Para fechá-lo, é preciso mostrar agora que o retórico já se baseia numa linguística: a "intuição" interpretativa repercute um prejuízo gramatical. Quando se tem detectado no sentido do enunciado, a existência de uma figura, a única forma é confrontá-lo com o que estimamos como seu "sentido literal" e constatar um intervalo entre eles. Porém, esse "sentido literal" não é nada mais que outa significação da frase completada por alguns aportes contextuais - ou antes completada pelo mínimo de aporte contextual necessário para obter um valor semântico comunicável (esta noção tão pouco definível como é de "contexto mínimo" é frequentemente utilizada de fato e, me parece necessária para quem trabalha com a ideia de um "sentido literal" do enunciado). Por isto, concluise facilmente que a interpretação por figuras retóricas pressupõe uma linguística da frase. Voltamos ao exemplo da lítotes. As gramáticas de Port-Royal obrigam a admitir que cada frase é constituída, semanticamente, pela aplicação de um "modus", único, e de um "dictum", igualmente único - com uma exceção para certas frases complexas, cuja significação é dita "composta". O dictum expressa uma ideia, uma representação do entendimento, enquanto o modus expressa a escolha, a atitude, ou em termos cartesianos, a vontade do locutor (ainda que tenha vários modus, todos serão por definição, atribuídos a um sujeito único, o locutor). Não é, então, possível, que dois pontos de vista opostos se expressem no enunciado de uma frase, e por conseguinte, não se pode ouvir no verso de Corneille uma voz do dever que diria "Eu te odeio". O sentido "literal", entendido como aquilo que se deriva imediatamente da frase, não poderia desse modo, ser polifônico: é n'est que la première moitié du cercle. Pour le boucler, il faut montrer maintenant que le rhétoricien s'appuie déjà sur une linguistique: 1' "intuition" interprétative répercute un préjugé grammatical. Quand on a à déceler, dans le sens d'un énoncé, l'existence d'une figure, le seui moyen est de le confronter avec ce qu'on estime son "sens littéral" et de constater un écart entre eux. Or, ce "sens littéral" n'est rien d'autre que la signification de la phrase, complétée par quelques apports contextuels - ou plutôt complétée par le minimum d'apport contextuel nécessaire pour obtenir une valeur sémantique communicable (cette notion si peu définissable soit-elle, de "contexte minimum" est souvent utilisée en fait, et me semble nécessaire à qui travaille avec l'idée d'un "sens littéral" de l'énoncé.) D'où l'on conclut facilement que l'interprétation par figures rhétoriques présuppose une linguistique de la phrase. Revenons à l'exemple de la litote. Les grammaires issues de PortRoyal obligent à admettre que chaque phrase est constituée, sémantiquement, par l'application d'un "modus", et d'un seul, à un "dictum", lui aussi unique avec une exception pour certaines phrases complexes, dont la signification est dite "composée". Le dictum exprime une idée, une représentation de l'entendement, alors que le modus exprime le choix, l'attitude, ou en termes cartésiens, la volonté du locuteur (même s'il y a plusieurs modus, ils seront tous, par définition, attribués à un sujet unique, le locuteur). Il n'est donc pas possible que deux points de vue opposés s'expriment dans l'énoncé d'une phrase, et on ne peut donc pas entendre, dans le vers de Corneille, une voix du devoir qui dirait "Je te hais". Le sens "littéral", entendu comme celui qui se dérive immédiatement de la phrase, ne saurait donc être polyphonique: c'est simplement 
simplesmente a atribuição à pessoa representada por eu de um predicado negativo "não odiar você". Por isso, a necessidade em seguida, de imaginar uma figura, por exemplo, uma lítotes, se se quer a partir deste valor de base, compreender a força tomada pelo enunciado no contexto em que ele está empregado. Neste momento, o círculo está fechado: o linguista deve se apoiar sobre interpretações retóricas para construir uma gramática que as explique, mas esta retórica, é em si mesma, já fundada sobre uma gramática.

Para finalizar, gostaria, somente, de formular de um modo compacto o que me parece ser a razão necessária do círculo que fingi me surpreender. Enquanto linguistas semanticistas, tomamos o sentido dos enunciados como meio para acessar a significação das frases, o critério utilizado para decidir a significação é antes de tudo, que ela deve dar conta do sentido. Mas esse critério apenas é justificável se esta significação pretende ser aquela de que se servem de fato os sujeitos falantes para interpretar os enunciados: podemos então sustentar que deixamos explícito, ao simular os efeitos, os conhecimentos semânticos que os sujeitos falantes possuem e que fazem funcionar sem sabê-lo. Mas é claro que os "valores imediatos" a partir dos enunciados no uso cotidiano, não são reconstruídos pelos ouvintes em termos de um só cálculo interpretativo fundado sobre significações de frase. Supondo até que sejamos capazes de gerar esses "valores imediatos" das significações atribuídas às frases, isto não comprovaria em nada, a seguir, que as nossas significações tenham uma realidade qualquer. $\mathrm{O}$ que precisamos então, buscar a simular, com a ajuda das significações, são somente interpretações padronizadas, l'attribution à la personne représentée par je d'un prédicat négatif "ne pas te haïr". D'où la nécessite, ensuite, d'imaginer une figure, par exemple une litote, si l'on veut, à partir de cette valeur de base comprendre la force prise par l'énoncé dans le contexte où il est employé. Cette fois-ci, le cercle est complet: le linguiste doit s'appuyer sur des interprétations rhétoriques pour construire une grammaire qui les explique, mais cette rhétorique est elle-même déjà fondée sur une grammaire.

Pour finir, je voudrais seulement formuler d'une façon compacte ce qui me semble la raison nécessaire du cercle dont j'ai fait semblant de m'étonner. En tant que linguistes sémanticiens, nous prenons le sens des énoncés comme moyen pour accéder à la signification des phrases, le critère utilisé pour décider de la signification étant avant tout qu'elle doit rendre compte du sens. Mais ce critère n'est justifiable que si cette signification prétend être celle dont se servent en fait les sujets parlants pour interpréter les énoncés: nous pouvons soutenir, alors que nous avons rendu explicite, en en simulant les effets, les connaissances sémantiques que les sujets parlants possèdent et mettent en œuvre sans le savoir. Mais il est clair que les "valeurs immédiates" à partir des énoncés dans l'usage quotidien ne sont pas reconstruites par les auditeurs au terme d'un calcul interprétatif fondé sur des significations de phrase. A supposer même que nous soyons capables de générer ces "valeurs immédiates" des significations attribuées aux phrases, cela ne nous prouverait par suite en rien que nos significations aient une quelconque réalité. Ce que nous devons donc chercher à simuler, à l'aide des significations, ce sont seulement des interprétations standardisées, idéalisées, 
racionalizadas. No entanto, estas são, infelizmente, construídas com a ajuda de conhecimentos implícitos de ordem (pseudo-) teórico, ou (pseudo-) científico. Fundar a gramática sobre tais interpretações, é, portanto, finalmente, fundá-la sobre si mesma. É possível romper, ou pelo menos expandir o círculo? É possível, em semântica linguística, dizer algo que não se reduza a esses "vérités de La Palisse" de que nos repreendia Michel Pêcheux? ${ }^{16} \mathrm{Eu}$ acho que sim, claro: caso contrário, eu reservaria para conversas de corredor desiludidas o que tentei proclamar. De qualquer forma, a primeira condição para sair de uma prisão (como dizia proximamente Sócrates para seus colegas de caverna) é não acreditar que estamos fora. rationalisées. Or, celles-ci sont, par malheur, construites à l'aide de connaissances implicites, d'ordre (pseudo-) théorique, ou (pseudo-) scientifique. Fonder la grammaire sur de telles interprétations, c'est donc finalement la fonder sur elle-même. Est-il possible de briser, ou au moins d'élargir le cercle? Est-il possible, en sémantique linguistique, de dire quelque chose qui ne se réduise pas à ces "vérités de $\mathrm{La}$ Palisse" que nous reprochait Michel Pècheux? Je le pense bien sùr: sinon je réserverais pour des conversations de couloir désabusées ce que j'ai essayé de proclamer. En tout cas, la première condition pour sortir d'une prison (comme disait à peu près Socrate à ses camarades de caverne), c'est de ne pas croire qu'on est dehors.

Artigo recebido em: dezembro de 2016.

Aprovado e revisado em: fevereiro de 2017.

Publicado em: abril de 2017

\section{Para citar este texto:}

DUCROT, Oswald. A interpretação em Semântica Linguística: um ponto de partida imaginário. [Trad. Bras. de L'interprétation en Sémantique Linguistique: un point de départ imaginaire]. Entremeios [Revista de Estudos do Discurso, on-line], Seção Estudos, Programa de Pós-Graduação em Ciências da Linguagem (PPGCL), Universidade do Vale do Sapucaí (UNIVÁS), Pouso Alegre (MG), vol. 14, p. 111-134, jan. - jun. 2017.

DOI: http://dx.doi.org/10.20337/ISSN2179-3514revistaENTREMEIOSvol14pagina111a134

\footnotetext{
${ }^{15}$ N.T: Em português, PÊCHEUX, M. Semântica e Discurso: uma crítica à afirmação do óbvio. Trad. Bras. 4ª ed. Campinas, SP: Editora Unicamp, 2009.

${ }^{16}$ Michel Pêcheux. Les Vérités de la Pallice. Maspero: Paris, 1975.
} 